\title{
¿Son eficaces para disminuir el acoso escolar los programas de alumno mediador de reciente implantación?
}

\section{Are recently implemented peer mediation programs effective in diminishing bullying?}

Fecha de recepción: 23-05-2021

Fecha de aceptación: 21-01-2022
Lidón Villanueva Badenes

Departamento de Psicología Evolutiva y de la Educación, Social y Metodología, Universitat Jaume I de Castellón

Inmaculada Usó Guiral

Departamento de Psicología Evolutiva, Social y Metodología (Facultad de Ciencias de la Salud) y Unidad de Apoyo Educativo (USE)

Universitat Jaume I de Castelló de la Plana.

Juan E. Adrián Serrano

Departamento de Psicología Evolutiva y de la Educación, Social y Metodología,

Universitat Jaume I de Castellón.

\section{resumen/alsstract:}

En este estudio se analiza la eficacia de los sistemas de alumno mediador como medida para disminuir las situaciones de acoso escolar, teniendo en cuenta el tiempo de implantación en el centro. Para ello se compara un centro con programa de mediación (desde hace dos cursos) y otro sin programa de intervención, en T1 (línea base) y T2 (tras la intervención). Los participantes fueron 331 estudiantes, de $1^{\circ}$ y $3^{\circ}$ de ESO (media $=13.4$ años), y las variables analizadas: actitudes pro-víctima, roles ante el acoso y clima de centro.

Los resultados indican que las actitudes pro-víctima y los roles ante el acoso no cambiaron a pesar de la intervención. Incluso pudieron observarse puntuaciones más bajas en clima escolar en el centro con programa de mediación, atribuible quizás, al efecto de sensibilización y toma de conciencia que se producen en los primeros momentos de implementación del mismo. Se ha demostrado la ausencia de resultados positivos en los dos primeros años de la puesta en marcha del programa, siendo importante para conseguirlos la consolidación a largo plazo de una cultura de mediación en todo el centro.

In this study, the effectiveness of a peer mediation program as a possible measure to diminish bullying behaviors is secondary education is analysed, taking into account time implementation in the center. Two centers are compared in Time 1 (baseline) and Time 2 (after program intervention), one with mediation program since two academic courses, and the other, with no mediation program. Participants were 331 students, from courses $1^{\circ}$ and $3^{\circ}$ ESO $($ mean $=13.4$ years), and the variables assessed: pro-victim attitudes, the roles adopted by the youngsters and the school climate.

Results showed that pro-victim attitudes and roles adopted did not change after program intervention. Even more, lower scores in school climate in T2 could be appreciated in the center with mediation program. Maybe this result is due to the effect of sensitivity and awareness about bullying than can appeared in the first phases of the intervention. In summary, this study offers the evidence of the lack of positive results in the first two years of program implementation, pointing out the importance of long-term consolidation of culture mediation in the centers.

\section{palabras clave/keywords:}

acoso escolar, alumno mediador, tiempo de implementación, clima de centro.

bullying, peer mediation, implementation time, school climate. 


\section{Introducción}

Según fuentes nacionales, se debe tener en cuenta que en el 35.5\% de las clases de Educación Primaria y Secundaria, el alumnado informa de la existencia de acoso escolar, siendo las formas más frecuentes, los insultos y motes (79.8\%), empujones, golpes y patadas (68.3\%), o el aislamiento (25.8\%), (Informe Mutua Madrileña y Fundación Anar, 2019). Estos datos son una razón más que suficiente para que se realicen acciones contra la intimidación, teniendo en cuenta las consecuencias que se derivan de estas situaciones.

Estas consecuencias existen no sólo para las víctimas, sino también para los jóvenes que intimidan y para los testigos del acoso escolar (Bradshaw, 2015; Husky et al., 2020; Menesini y Salmivalli, 2017; Sánchez et al., 2012). Las víctimas sufren por el acoso al que son sometidos por sus iguales, en términos de desarrollo social, emocional, académico y físico (Copeland et al., 2014; Tofti et al., 2011), mientras que los agresores adquieren conductas antisociales, que pueden implicar consecuencias negativas para el resto de sus vidas (Cerezo y Méndez, 2013), sobre todo a través de mecanismos de desvinculación emocional (Lo Cricchio et al., 2021; Romera et al., 2021). Al tiempo, se producen efectos negativos tanto en el clima de centro como en los profesionales de la educación, en particular, y en toda la comunidad educativa en general (Lane-Garon y Richardson, 2003).

\section{Sistemas de apoyo entre iguales}

Gracias a los sistemas de apoyo entre iguales en general, y a los sistemas de mediación en particular, en donde los iguales se convierten en agentes de cambio, en soporte y ayuda para el resto, se pueden establecer estrategias globales que permiten reducir el impacto de los conflictos más habituales de convivencia (García-Raga et al., 2019; Lee et al., 2013; Silva et al., 2021). De esta manera, se promueve que el alumnado se involucre en la participación del programa de forma directa (Andrés, 2007; Tirado y Conde, 2016). Estos programas, además cuentan con una buena eficacia en los primeros cursos de la Educación Secundaria Obligatoria (del Barrio et al., 2011; Gaffney et al., 2021b; Yeager et al., 2015), puesto que el paso a esta nueva etapa educativa, sobre todo entre los 11 y los 13 años, está marcado por la mayor utilización del acoso relacional directo e indirecto, la persecución psicológica y las formas más indirectas del acoso (Romera et al., 2017), y por tanto, es donde más sentido tiene que la ayuda entre iguales se establezca de modo formal en los centros. También han mostrado su eficacia en las redes sociales (Banerjee et al., 2012) o mediante la prestación de asesoramiento en grupos de discusión sobre qué hacer ante el acoso cibernético (DiBasilio, 2008).

En general, para conocer el grado de eficacia conseguido por estos sistemas de apoyo entre iguales, existen tres niveles de variables indicadoras de posibles cambios: individual (actitudes pro-víctima), grupal (roles adoptados ante el acoso), y organizacional (clima de centro). En este trabajo se tendrán en cuenta todos estos niveles. Según diferentes estudios, existe relación entre las conductas de desarrollo moral y las actitudes adoptadas en los diferentes grupos, que pueden explicar los roles que se asumen en las situaciones de bullying (Bauman et al., 2020; Tognetta et al., 2016). Así, no todos los estudiantes adoptan un papel fijo en las situaciones de bullying (agresor, seguidor, víctima, defensor de la víctima y pú- 
blico), de hecho, tras las intervenciones de prevención o intervención, se pueden producir cambios en estas clasificaciones.

También tras la intervención con sistemas de apoyo entre iguales, las actitudes en favor de la víctima pueden verse potenciadas, así como los roles de defensor de la víctima y del espectador (Salmivalli et al., 2005). Pero puede que no se produzca una correlación entre las actitudes de un estudiante y el comportamiento real que adopta en presencia del grupo (Salmivalli et al., 1996). Menesini et al. (2003) describieron la asunción de roles tanto en el grupo control como experimental. Después de la intervención de un programa de ayuda entre iguales y en el grupo control, los roles negativos y público se habían incrementado, mientras que las actitudes pro-víctima, disminuían. Del mismo modo, en un estudio de Usó et al. (2016), comparando un centro con programa alumno mediador, con una trayectoria de implantación consolidada de cuatro cursos, y un centro sin programa de intervención, se encontró que en el centro con programa, las actitudes de defensa de la víctima aumentaron entre el alumnado y mejoró el clima escolar, mientras que en el centro sin programa, el rol negativo de seguidor/a-ayudante del agresor aumentó y el rol positivo de defensor de la víctima disminuyó.

No han sido pocos los estudios que han concluido que, tras la intervención con sistemas de apoyo entre iguales, el clima de centro se percibe de manera más positiva y segura (Barruel y Nissanka, 2021; Lane-Garon y Richardson, 2003; Usó et al., 2016), y además la frecuencia y la intensidad de la intimidación disminuye (Palladino et al., 2012). Pero se debe tener en cuenta que cambiar el clima de centro cuando se percibe como negativo, es una tarea compleja con resultados a medio y largo plazo, y en pocas ocasiones se obtienen evidencias inmediatas. Además, conseguir este propósito requiere un esfuerzo y compromiso de toda la comunidad educativa y también de las familias (Galindo y Sanahuja, 2021).

La mayoría de los estudios se han centrado en comprobar la eficacia e implicaciones de los sistemas de apoyo entre iguales, y lo han hecho en centros con larga trayectoria de implantación de los programas y que ya cuentan con la institucionalización y conocimiento de estos por parte de todos los miembros de la comunidad educativa (Andrés, 2007; Andrés y Gaymard, 2014; Usó et al., 2016). Pero en el caso de los centros que se deciden a implementar estos programas en sus centros, ¿cuánto tiempo debe transcurrir para apreciar el impacto de los mismos en la comunidad educativa? Torrego y Galán (2008) plantean que es necesario un cambio de mentalidad en el tratamiento de los conflictos, ya que su resolución es una cuestión lenta, que requiere tiempo para que realmente consoliden los efectos de los programas. En este sentido, algunos autores han indicado que, para la consolidación de un programa de mediación escolar, la media es de tres años (Boqué, 2004) y otros han apuntado que los programas con menos de dos años de trayectoria en los centros tienen pocas probabilidades de contribuir de forma completa a mejorar el clima de centro (Houlston y Smith, 2009). Incluso algunos autores han indicado que puede haber un aumento de los conflictos en las primeras etapas de implementación de los programas en los centros (Andrés, 2007; Houlston et al., 2009; Ortega et al., 2004; Smith, 2003).

Por todo ello, y dada la gran incidencia del acoso escolar, y sus consecuencias negativas sobre los implicados, el presente estudio tiene como objetivo conocer si existen cambios en 
las diferentes variables implicadas en los conflictos entre el centro en el que se desarrolla de manera reciente un programa alumno mediador (en su segundo año), respecto al centro en el que no se realiza ninguna intervención directa de mejora de la convivencia. Para ello, se plantean las siguientes hipótesis: en el centro con programa de mediación se producirá una mejora en las actitudes pro-víctima, al tiempo que las puntuaciones en roles positivos o defensores de la víctima aumentarán. Finalmente, modificar el clima de centro es una tarea difícil y costosa en el tiempo, por tanto, dada la poca trayectoria del programa de intervención entre iguales, en esta variable se espera no encontrar cambios significativos entre los centros.

Este objetivo se acompaña de las siguientes contribuciones propias del trabajo, que intentan superar limitaciones de estudios previos: un análisis de medidas repetidas en el tiempo, tal como aconsejan los expertos (Gaffney et al., 2021a), incluyendo diferentes tipos de medidas de cambio (individual, grupal y contextual), así como diferentes tipos de información (autoinforme e iguales). Por último, el trabajo incorpora un grupo control que no pertenece al contexto del grupo de intervención (centros escolares diferentes).

\section{Método}

\section{Participantes}

Los participantes fueron 331 alumnos de dos centros de Educación Secundaria Obligatoria de la misma localidad ( $1^{\circ}$ y $\left.3^{\circ} \mathrm{ESO}\right)$. Los centros, seleccionados a través de un muestreo de conveniencia, se han elegido porque presentan características similares, tanto a nivel geográfico, como socioeconómico y de oferta académica. En segundo lugar, porque en uno de ellos estaba instaurado en el centro por segundo año consecutivo, un programa de alumno mediador (centro B), con 179 estudiantes (54.1\%). Por su parte, el otro centro no cuenta con ningún programa de ayuda entre iguales (centro A), con 152 estudiantes $(45.9 \%)$.

En el centro A, 80 estudiantes (52.6\%) eran de primer curso, mientras que $72(47.4 \%)$ se encontraban cursando $3^{\circ}$ de la ESO (ver Tabla 1). En el centro B, 80 estudiantes (44.7\%) pertenecían a los cursos de $1^{\circ} \mathrm{ESO}$, mientras que los 99 restantes $(55.3 \%)$ a $3^{\circ}$ de $\mathrm{ESO}$. Se escogieron los estudiantes de primero y tercero, puesto que los de segundo curso contaban con una gran carga de exámenes tanto internos como externos.

Tabla 1.- Distribución de la muestra total por centros

\section{Centro}

\begin{tabular}{lccc} 
& Sin programa $(\mathrm{A})$ & Con programa $(\mathrm{B})$ & TOTAL \\
\hline Estudiantes & $152(45.9 \%)$ & $179(54.1 \%)$ & 331 \\
Media de edad & $13.5 \mathrm{años}$ & $13.4 \mathrm{años}$ & 13.4 años \\
Estudiantes de primer curso & $80(52.6 \%)$ & $72(47.4 \%)$ & $160(48.3 \%)$ \\
Estudiantes de tercer curso & $80(44.7 \%)$ & $99(55.3 \%)$ & $171(51.7 \%)$ \\
Chicos & $85(25.7 \%)$ & $87(26.3 \%)$ & $172(52 \%)$ \\
Chicas & $67(20.2 \%)$ & $92(27.8 \%)$ & $159(48 \%)$ \\
\hline
\end{tabular}


Al comparar los dos centros, se puede observar que no hay diferencias en la distribución por sexo $\left(\chi^{2}=1.76 ; \mathrm{p}=.18\right),(172$ chicos y 159 chicas $)$, ni en la edad media de los estudiantes (media del centro $\mathrm{A}=13.5$, media del centro $\mathrm{B}=13.4 ; \mathrm{t}=1.037 ; \mathrm{p}=.30$ ).

\section{Instrumentos}

Escala de Roles “Adivina Quién” (Salmivalli et al., 1996), adaptada por Sutton y Smith (1999). Es un cuestionario compuesto por 21 ítems, en el cual el estudiante debe nominar siguiendo el método propuesto por Camodeca y Goossens (2005). Hay que identificar a través de la pregunta ¿Adivina quién?, qué estudiantes de la clase encajan en cada rol, siempre que la puntuación estandarizada de los ítems que hacen referencia a ese rol sea más alta que la media de la clase y más alta que en ningún otro rol. La única excepción la constituye el rol de víctima, donde un alumno pertenece si es nominado por al menos el 20\% de la clase. Diversos autores (Sutton y Smith, 1999) agrupan a los ayudantes y animadores del agresor en un nuevo rol, los seguidores. Esta agrupación se ha realizado también en este trabajo. Los roles analizados son los siguientes: El "agresor" es la persona que realiza las acciones de humillación y acoso contra la víctima ("empieza a atacar o a molestar"), el "animador" indica acciones para que el agresor le haga a la víctima ("le dice cosas al agresor como demuéstrale quien manda aquî"), el "ayudante" refuerza la conducta del agresor ("llama a otros para que vean la agresión"), el "defensor" apoya a la víctima y trata de parar el acoso ("le cuenta a una adulto que se están metiendo con alguien"), el "espectador" observa la agresión, y colabora con el acoso, por no denunciarlo a los adultos ("finge no darse cuenta de lo que pasa") y, por último, la "víctima" ("siempre se meten con él/ella"). En el estudio original (Salmivalli et al., 1996), la escala demostró propiedades psicométricas adecuadas (Agresor $\alpha=.93$; Ayudante $\alpha=.81$; Animador $\alpha=.91$; Defensor $\alpha=.93$; Espectador $\alpha=$ .89). Lo mismo ocurrió en el presente estudio, en el que obtuvieron los siguientes datos: T1, Agresor $\alpha=.89$; Seguidor $\alpha=.81$; Defensor $\alpha=.75$; Espectador $\alpha=.78$; T2, Agresor $\alpha=$ .84 ; Seguidor $\alpha=.80$; Defensor $\alpha=.76$; Espectador $\alpha=.79$.

Escala de Actitudes Pro-víctima (Rigby y Slee, 1991). En esta escala, se evalúan las actitudes pro-víctima. Los estudiantes deben responder ("de acuerdo", "no sé" "en desacuerdo"), según las afirmaciones de los 10 ítems que representan situaciones de acoso. Cada respuesta tiene asociada una puntuación que puede ser de 1,2 ó 3 puntos, que se asignan según el ítem sea directo o inverso. La puntuación total oscila de 10 a 30 puntos, siendo las puntuaciones más elevadas las que demuestran una actitud más favorable en defensa de la víctima. Algunos de los ítems que se plantean son los siguientes: "Es gracioso ver cómo se ofenden algunos chicos cuando les molestan", "los chicos blandos me ponen enfermo", "me gusta cuando alguien se pone de parte de los chicos que son molestados/agredidos". La escala original demostró buena fiabilidad y validez ( $\alpha=.78 ; 3$ factores que explicaban el $34 \%$ de la varianza), (Rigby y Slee, 1991). En la misma línea, el presente estudio obtuvo un $\alpha=.71$ (T1), y un $\alpha=.77$ (T2).

Cuestionario sobre Clima de Centro (Ortega y Del Rey, 2003). El instrumento permite conocer la opinión de los estudiantes sobre problemas de convivencia en el centro, las relaciones que mantienen los compañeros, el profesorado y cómo ven la participación de las familias 
en la vida del centro. Solo 14 de los 33 ítems que componen este cuestionario, se han incluido en este estudio (en concreto, aquellos que presentaban la misma escala likert). Algunos ejemplos de ítems son “¿Cómo te llevas con tus compañeros?”, “¿Qué opinión crees que tienen de ti tus profesores?", "Hay enfrentamientos alumno-profesor" ó "Los alumnos se pelean". Los estudiantes deben indicar si las situaciones se dan en el centro "nada", "poco", "regular" o "mucho". Las respuestas de alternativa múltiples se cuantifican en puntuaciones de 0 a 4 , siendo positivas las puntuaciones más elevadas en los ítems directos.

\section{Procedimiento}

Para realizar este estudio de medidas repetidas en el tiempo (pretest-postest), se solicitó permiso a la Conselleria de Educación, informando del objetivo de la investigación, así como del procedimiento a seguir (código Comité Ético 3/2010). Asimismo, se les facilitaron autorizaciones a los tutores de los grupos de primero y tercer curso de ESO, para que los padres y madres que lo consideraran oportuno dieran su consentimiento para que sus hijos participaran en el estudio.

A los alumnos autorizados, se les explicó el significado de "acoso entre iguales e intimidación" y el procedimiento para responder a los tres cuestionarios, así como el principio de confidencialidad de sus respuestas. Los cuestionarios se administraron en la clase de tutoría, de manera grupal. Para poder comparar los resultados, se recabó información en dos momentos del curso escolar: Tiempo 1 (mes de diciembre) y Tiempo 2 (mes de junio, tras la intervención con el programa de ayuda entre iguales). Cabe decir que la intervención con el sistema de apoyo entre iguales fue coordinada y realizada por el equipo directivo y claustro de profesores del centro, siguiendo las indicaciones del Modelo de Resolución de Conflictos Escolares (Torrego, 2005).

\section{Análisis de datos}

Para los análisis intergrupo se ha utilizado el Análisis Multivariado de la Varianza (MANOVA) con la variable centro (con o sin intervención) como variable independiente y las variables roles, actitudes y clima de centro, como variables dependientes. Para los análisis intragrupo, se han realizado pruebas $T$-Student para muestras relacionadas, calculando el tamaño del efecto.

\section{Resultados}

\section{Análisis intergrupo}

En el tiempo 1, para las variables roles y actitudes pro-víctima, el efecto de la variable centro (con o sin intervención) no tuvo resultados significativos (Traza de Pillai $=.025, p$ $=.139$ ). Y, por tanto, se apoya la afirmación de que los dos centros parten de una situación parecida, sin diferencias en las variables analizadas. Esto permitirá conocer los cambios que se han producido en el segundo tiempo.

También para el tiempo 1 se analizó el efecto de la variable centro (con o sin intervención) sobre el clima escolar, y en este caso sí aparecían diferencias significativas (Traza de Pillai $=.220, p=.000$ ). Por tanto, al comparar el primer tiempo en ambos centros, se asumen 
situaciones iniciales diferentes respecto al clima de centro (ver Tabla 2). En el centro sin programa de mediación, los compañeros se llevan mejor entre ellos y existen menos diferencias en las normas de clase de los profesores. Sin embargo, en el centro con programa de mediación, los padres participan más en las actividades del centro, se respetan más las normas, los profesores van menos "a lo suyo" y tratan de entender más a los alumnos. Asimismo, los estudiantes del centro con programa de mediación indican con mayor frecuencia abusos por parte de los compañeros que en el centro sin programa de mediación.

Tabla 2.- Efecto de la variable centro (con o sin programa) en la variable clima escolar en tiempo 1

\begin{tabular}{lcccc}
\hline & \multicolumn{2}{c}{$\mathrm{M}(\mathrm{DT})$ en Centro } & \\
\cline { 2 - 3 } Clima escolar en tiempo 1 & Sin programa & $\begin{array}{c}\text { Con } \\
\text { programa }\end{array}$ & \\
\hline Cómo te llevas con los compañeros & $2.69(.591)$ & $2.85(.480)$ & 7.410 & $.007^{* *}$ \\
Hay diferentes normas en clase según el profesor & $1.00(.503)$ & $1.25(.754)$ & 11.663 & $.001^{* *}$ \\
Los padres participan en las actividades del & $1.42(.867)$ & $1.80(.851)$ & 15.638 & $.000^{* *}$ \\
centro & & & & \\
No se respetan las normas & $1.86(.825)$ & $1.63(.820)$ & 6.389 & $.012^{* *}$ \\
Los profesores van a lo suyo & $1.28(1.084)$ & $1.04(1.024)$ & 4.228 & $.041^{*}$ \\
Los profesores no entienden a los alumnos & $1.91(.938)$ & $1.66(1.028)$ & 5.444 & $.020^{*}$ \\
Cúantas veces te han maltratado los compañeros & $.17(.412)$ & $.32(.536)$ & 8.072 & $.005^{* *}$ \\
\hline
\end{tabular}

*Nivel de significación .05 **Nivel de significación .01

Tras los análisis realizados en el segundo tiempo, se puede comprobar que no existen diferencias significativas de la variable centro (con o sin intervención) sobre los roles y las actitudes pro-víctima (Traza de Pillai $=.013, p=.515$ ).

Sin embargo, también en este segundo tiempo, aparece el efecto significativo de la variable centro (con o sin intervención) sobre el clima de centro, como se muestra en la Tabla 3 (Traza de Pillai $=.323, p=.000$ ). En el centro con programa de mediación, tras la intervención, el clima de centro no mejora, puesto que los estudiantes piensan que continúan existiendo diferencias en las normas de clase según los profesores, más enfrentamientos entre alumnoprofesor, más alumnos que se insultan, se pelean y dicen malas palabras en clase. Los estudiantes sienten que los compañeros los maltratan con mayor frecuencia que en el centro sin intervención, al igual que reciben más ciberbullying e intimidaciones. Por último, en el centro sin programa, los estudiantes continúan pensando que los profesores los entienden menos que en el centro con programa de mediación. 
Tabla 3.- Efecto de la variable centro (con o sin programa) en la variable clima escolar en tiempo 2

\begin{tabular}{lcccc}
\hline \multirow{2}{*}{ Clima escolar en tiempo 2 } & \multicolumn{2}{c}{$\mathrm{M}(\mathrm{DT})$ en Centro } & \\
\cline { 2 - 4 } & Sin programa & Con programa & & \\
\hline Hay diferentes normas en clase según profesor & $1.00(.503)$ & $1.37(.678)$ & 31.386 & $.000^{* *}$ \\
Hay enfrentamientos alumno-profesor & $.92(.753)$ & $1.43(.867)$ & 31.873 & $.000^{* *}$ \\
Hay malas palabras en clase & $1.81(.867)$ & $2.02(.909)$ & 4.228 & $.041^{*}$ \\
Los alumnos se insultan & $1.65(.957)$ & $1.99(.896)$ & 11.310 & $.001^{* *}$ \\
Los alumnos se pelean & $.84(.825)$ & $1.53(1.029)$ & 43.946 & $.000^{* *}$ \\
Los profesores no entienden a los alumnos & $1.99(.894)$ & $1.72(.972)$ & 6.604 & $.011^{*}$ \\
Cúantas veces te han maltratado compañeros & $.16(.433)$ & $.33(.549)$ & 9.573 & $.002^{* *}$ \\
Has recibido ciberbullying & $.02(.140)$ & $0.08(.326)$ & 4.186 & $.042^{*}$ \\
Frecuencia con que se dan intimidaciones en & $1.02(5.35)$ & $1.28(.583)$ & 18.251 & $.000^{* *}$ \\
el centro & & & & \\
\hline
\end{tabular}

*Nivel de significación .05 **Nivel de significación .01

\section{Análisis intragrupo}

A continuación, se presentan los análisis intragrupo para las variables roles, actitudes y clima de centro, con el fin de conocer las posibles diferencias en cada centro, de un tiempo a otro. Ni en el centro A ni en el centro B, existen diferencias significativas en la distribución de roles participantes de un tiempo al otro (obteniendo significaciones que oscilan entre .33 y .97). Respecto a la variable actitudes, tampoco en ninguno de los dos centros existen diferencias significativas del tiempo 1 al tiempo 2 (centro $\mathrm{A}, \mathrm{M} 1=25.59$; $\mathrm{M} 2=25.48, p=$ .566. Centro B, M1 = 26.37; M2 = 26.49, $p=.669$ ).

Por último, dentro de la variable clima de centro, los resultados indican que en el centro A (sin programa de mediación), se han obtenido diferencias significativas entre el tiempo 1 y 2 en los ítems que se detallan en la Tabla 4, todos ellos, con un pequeño tamaño del efecto. Los estudiantes consideran que, pasado el curso, sus compañeros tienen una mejor opinión de ellos mismos, y que los padres participan más en las actividades del centro respecto al tiempo 1. Incluso, aún sin intervención, se produce una mejora respecto a los enfrentamientos entre alumnos y profesores, los alumnos se pelean menos, y disminuyen las situaciones de ciberbullying y la frecuencia de las intimidaciones.

En el centro B (con programa de mediación), han resultado significativos dos ítems relacionados con el clima de centro, que se indican en la Tabla 5. La pregunta sobre la relación que se tiene con los compañeros, indica que los estudiantes en el tiempo 2 se llevan peor con sus compañeros de clase, respecto al tiempo $1(\mathrm{t}=3.210, p=.002$, $\mathrm{D}$ de Cohen $=.34, \mathrm{r}=.16)$. También hay diferencias en el ítem en el que se pregunta si se producen discusiones entre 
Tabla 4.- Diferencias intragrupo del tiempo 1 al 2 en los ítems en clima escolar para el centro A (sin programa de mediación)

\begin{tabular}{lccccccc}
\hline & tiempo 1 & tiempo 2 & & & & \\
\cline { 2 - 4 } Centro A & $\mathrm{M}(\mathrm{DT})$ & $\mathrm{M}(\mathrm{DT})$ & $\mathrm{T}$ & $\mathrm{p}$ intragrupo & $\mathrm{D}$ & $r$ \\
\hline $\begin{array}{l}\text { Qué opinión crees que tienen de tí tus } \\
\text { compañeros }\end{array}$ & $2.31(.712)$ & $2.47(.574)$ & -2.164 & $.032 *$ & -.039 & -.019 \\
$\begin{array}{l}\text { Los padres participan en la vida del } \\
\text { centro }\end{array}$ & $1.42(.865)$ & $1.70(.631)$ & -3.245 & $.001 * *$ & -.369 & -.181 \\
$\begin{array}{l}\text { Hay enfrentamientos alumnos } \\
\text { profesores }\end{array}$ & $1.18(.831)$ & $.92(.751)$ & 3.163 & $.002 * *$ & .328 & .167 \\
$\begin{array}{l}\text { Los alumnos se pelean } \\
\text { Has recibido ciberbullying }\end{array}$ & $1.24(.919)$ & $.84(.823)$ & 4.429 & $.000 * *$ & .458 & .223 \\
Hay frecuentes intimidaciones & $.08(.294)$ & $.02(.140)$ & 2.358 & $.020 *$ & .260 & .129 \\
& $1.18(.520)$ & $1.02(.533)$ & 2.960 & $.004 * *$ & .303 & .150 \\
\hline
\end{tabular}

*Nivel de significación .05 **Nivel de significación .01

Tabla 5.- Diferencias intragrupo del tiempo 1 al 2 en los ítems en clima escolar para el centro B (con programa de mediación)

\begin{tabular}{lcccccc}
\hline & tiempo 1 & tiempo 2 & & & & \\
\cline { 2 - 3 } Centro B & $\mathrm{M}(\mathrm{DT})$ & $\mathrm{M}(\mathrm{DT})$ & $\mathrm{T}$ & $\mathrm{p}$ intragrupo & $\mathrm{D}$ & $r$ \\
\hline Cómo te llevas con tus compañeros & $2.85(.480)$ & $2.66(.637)$ & 3.210 & $.002 *$ & .034 & .016 \\
$\begin{array}{l}\text { Hay enfrentamientos entre alumnos y } \\
\text { profesores }\end{array}$ & $1.13(.864)$ & $1.43(.867)$ & -3.209 & $.002 *$ & -.35 & -.17 \\
\hline
\end{tabular}

*Nivel de significación .05 **Nivel de significación .01

alumnos y profesores $(\mathrm{t}=-3.209, p=.002, \mathrm{D}$ de Cohen $=-.35, \mathrm{r}=-.17)$. En esta ocasión los estudiantes indican que, tras el paso del tiempo, hay más enfrentamientos entre alumnos y profesores, que al inicio de curso.

\section{Discusión}

El objetivo del estudio fue el de evaluar el posible impacto de un programa de alumno mediador de reciente implantación, en los tres niveles de variables indicadoras de posibles cambios: individual (actitudes), grupal (rol) y organizacional (clima de centro), comparando dos institutos de Educación Secundaria. Uno contaba con un programa de alumno mediador de reciente implantación en su segundo año, y el otro no realizaba ningún tipo de intervención específica como medida de prevención de conflictos. Los resultados encontrados, tanto de los análisis intergrupo como intragrupo, no apoyan la primera hipótesis 
planteada en la que se esperaban mejoras de un tiempo a otro, en el centro con intervención, para las variables actitudes y roles.

En este sentido, los resultados obtenidos a nivel intergrupo en el tiempo 1, no muestran diferencias en las variables roles y actitudes, lo que indica una situación inicial similar para los dos centros. En el tiempo 2, la intervención planteada en el programa de alumno mediador de reciente implantación no parece haber tenido ningún efecto sobre las variables roles y actitudes, en contra de lo hallado en estudios anteriores (Menesini et al., 2003; Palladino et al., 2012; Usó et al., 2016). Sin embargo, en este caso concreto se trata de un programa de reciente implantación y, por tanto, no consolidado en el centro.

Respecto a la variable clima escolar, no se apoya la hipótesis inicial, que presuponía que no existirían diferencias significativas entre los centros tras la intervención, debido a la poca trayectoria del programa en el centro con intervención. En realidad, sí existen diferencias significativas entre centros. En concreto, en el tiempo 2, en el centro sin programa de intervención, se puede encontrar una mejora en el clima de centro respecto al tiempo 1. Los alumnos afirman que ha mejorado la opinión que tienen de ellos los compañeros, los padres participan más en la vida del centro, se dan menos peleas entre alumnos y profesores y entre los propios alumnos, y se ha reducido la frecuencia de intimidaciones en el centro, así como de ciberbullying. Por tanto, en este caso existen diferencias significativas a favor del centro sin programa de intervención, en el cual paradójicamente mejora el clima de centro, a pesar de no contar con ninguna estrategia de intervención explícita.

Pero ¿por qué en el centro con el programa alumno mediador no se aprecia ninguna mejora, e incluso, empeoran algunos aspectos del clima de centro en el tiempo 2? Según diferentes estudios, programas con menos de dos o tres años de trayectoria, no es probable que contribuyan de forma completa a mejorar todo lo que comporta el clima de centro, como la relación entre los compañeros, con el profesorado, entre otros aspectos (Boqué, 2004; Houlston y Smith, 2009). De hecho, algunos estudios encontraron que, tras un período de cuatro años de puesta en marcha del programa, algunos aspectos puntuales de las relaciones profesor-alumno no habían mejorado (Usó et al., 2016). Se debe considerar, por tanto, que la eficacia de los programas contra el bullying mejora cuando éstos se desarrollan de forma continua, a largo plazo, más que como una intervención puntual o a corto plazo (Carney y Merrell, 2001).

En este caso, el clima del centro con programa de mediación ha empeorado según los alumnos, que afirman que se llevan peor con los compañeros y que han aumentado los enfrentamientos alumno-profesor respecto al primer tiempo. Esto puede explicarse según algunos autores (Andrés, 2007; Salmivalli et al., 2005), por el hecho de que el clima de centro empeora tras la puesta en marcha de un programa, debido a un bajo grado de implicación de los agentes, o simplemente, porque al trabajar estos temas, el alumnado es más consciente de la presencia de situaciones de intimidación y acoso escolar y, por lo tanto, informa más de ello en las respuestas de los cuestionarios. De hecho, distintos autores (Cowie y Wallace, 2000; Houlston et al., 2009; Smith y Watson, 2004), indican que puede incluso haber un aumento de los conflictos de manera que no se produzcan las mejoras esperadas. 
Este estudio no se encuentra exento de limitaciones. En primer lugar, la falta de análisis de las propiedades psicométricas del instrumento de clima de centro en el presente estudio es una debilidad del mismo. Asimismo, hubiese sido interesante involucrar a todo el personal de los centros y a las familias en las actividades del programa de alumno mediador (Bradshaw, 2015) y, por tanto, analizar sus respuestas en los ítems relacionados con el clima escolar. En este sentido, la evaluación del clima de centro necesita ser reforzada con el objetivo de que se capturen todos sus matices, así como que se cubra con un mayor número de informantes. Por otra parte, otra limitación del presente estudio es no haber incluido elementos de carácter más objetivo en la evaluación del impacto del programa como, por ejemplo, número de conflictos, casos de acoso notificados, partes de faltas por peleas, expulsiones por temas de acoso, etc.

Aún así, los resultados obtenidos evidencian la ausencia de mejora en los primeros años de implantación del programa de alumno mediador, como medida de disminución de las situaciones de acoso escolar. Por lo tanto, los usuarios y agentes de este tipo de programas deben ser conscientes de este hecho para evitar el abandono temprano de la intervención, sabiendo que la eficacia de estos se logra a largo plazo, transcurrido un tiempo desde la puesta en marcha en el centro. Una vez realizado esto de manera constante por todos los implicados en los programas, afortunadamente, la eficacia de los programas anti-bullying no parece disminuir en el tiempo (Fraguas et al., 2021).

\section{Referencias}

Andrés, S. (2007). Los sistemas de ayuda entre iguales como instrumentos de mejora de la convivencia en la escuela: evaluación de una intervención [Tesis doctoral, Universidad Autónoma de Madrid]

Andrés, S., \& Gaymard, S. (2014). The perception of school climate in two secondary schools during the implementation of a peer support program. Journal of Research in Educational Psychology, 12(2), 509-540. https://doi.org/10.25115/ejrep.33.13052

Banerjee, R., Robinson, C., \& Smalley, D. (2012). Evaluation of the beat bullying peer mentoring programme. University of Sussex.

Barruel, C. A., \& Nissanka, M. (2021). Examining the drivers of success in the Peace Foundation Aotearoa/New Zealand peer-mediation programs. In Information Resources Management Association (Ed), Research anthology on school shootings, peer victimization, and solutions for building safer educational institutions (pp. 779-801). IGI Global.

Bauman, S., Yoon, J., lurino, C., \& Hackett, L. (2020). Experiences of adolescent witnesses to peer victimization: The bystander effect. Journal of School Psychology, 80, 1-14. https://doi.org/10.1016/j.jsp.2020.03.002

Boqué, M.C. (2004). Mediación escolar: unidos ante el conflicto. Perspectiva CEP, 8, 55-69.

Bradshaw, C.P. (2015). Translating research to practice bullying prevention. American Psychologist Association, 70(4), 344-353. https://doi.org/10.1037/a0039114

Camodeca, M., \& Coossens, F.A. (2005). Children's opinions on effective strategies to cope with bullying: The importance of bullying role and perspective. Educational Research, 47, 93-105. https://doi. org/10.1080/0013188042000337587 
Carney, A., \& Merrell, K. (2001). Bullying in schools. Perspectives on understanding and preventing an international problem. School Psychology International, 22(3), 364-382. https://doi.org/10.1177/0143034301223011

Cerezo, F. \& Méndez, I. (2013). Agresores en bullying y conductas antisociales. European Journal of Investigation in Health, Psychology and Education, 3(7), 5-14. https://doi.org/10.30552/ejihpe.v3i1.32

Copeland, W.E., Wolke, D., Lereya, S.T., Shanahan, L., Worthman, C., \& Costello, E.J. (2014). Childhood bullying involvement predicts low-grade inflammation into adulthood. Proceedings of the National Academy of Sciences, 111, 7570-7575. https://doi.org/10.1073/pnas.1323641111

Cowie, H., \& Wallace, P. (2000). Peer support in action. From standing by to stand by. Sage Publications.

del Barrio, C., Barrios, Á., Granizo, L., van der Meulen, K., Andrés, S., \& Gutiérrez, H. (2011). Contribuyendo al bienestar emocional de los compañeros: Evaluación del Programa Compañeros Ayudantes en un instituto madrileño. European Journal of Education and Psychology, 4, 5-17. [fecha de Consulta 23 de mayo de 2021]. ISSN: 1888-8992. Disponible en: https://www.redalyc.org/articulo.oa?id=129318734001

DiBasilio, A. (2008). Reducing bullying in middle school students through the use of peer leaders. (Unpublished Master Thesis): University of Chicago: Illinois.

Fraguas, D., Díaz-Caneja, C. M., Ayora, M., Durán-Cutilla, M., Abregú-Crespo, R., Ezquiaga-Bravo, I., Martín-Babarro, J. \& Arango, C. (2021). Assessment of school anti-bullying interventions: a meta-analysis of randomized clinical trials. JAMA Pediatrics, 175(1), 44-55. https://doi:10.1001/jamapediatrics.2020.3541

Gaffney, H., Ttofi, M. M., \& Farrington, D. P. (2021a). Effectiveness of school-based programs to reduce bullying perpetration and victimization: An updated systematic review and meta-analysis. Campbell Systematic Reviews, 17(2), e1143.

Gaffney, H., Ttofi, M. M., \& Farrington, D. P. (2027b). What works in anti-bullying programs? Analysis of effective intervention components. Journal of School Psychology, 85, 37-56. https://doi.org/10.1016/j. jsp.2020.12.002

Galindo, E. I., \& Sanahuja, A. (2021). Implantación de un proyecto de mediación entre iguales: hacia una mejora de la convivencia en el contexto educativo. Márgenes, Revista de Educación, Universidad Málaga, 2(7), 130-147. https://doi.org/10.24310/mgnmar.v2i1.8459

García-Raga, L., Boqué, M. C., \& Grau-Vidal, R. (2019). Valoración de la mediación escolar a partir de la opinión de alumnado de educación secundaria de Castellón, Valencia y Alicante (España). Profesorado. Revista de Currículum y Formación del Profesorado, 23(1), 103-119. https://doi.org/10.30827/profesorado. v23i1.9146

Houlston, C., \& Smith, P.K. (2009). The impact of a peer counseling scheme the address bullying in an all-girl London secondary school: A short-term longitudinal study. British Journal of Educational Psychology, 79, 69-86. DOI: 10.1348/000709908X293878

Houlston, C., Smith, P.K., \& Jessel, J. (2009). Investigating the extent and use of peer support initiatives in English schools. Educational Psychology, 29(3), 325-344. https://doi.org/10.1080/01443410902926751

Husky, M. M., Delbasty, E., Bitfoi, A., Carta, M. G., Goelitz, D., Koç, C., Lesinskiene, S., Mihova, Z., Otten, R. \& Kovess-Masféty, V. (2020). Bullying involvement and self-reported mental health in elementary school children across Europe. Child Abuse \& Neglect, 107, 104601. DOI: https://doi.org/10.1016/j.chiabu. 2020.104601

Informe Informe Mutua Madrileña y Fundación Anar (2019). La opinión de los estudiantes. II Informe de Prevención del Acoso Escolar en Centros Educativos. Mutua Madrileña y Fundación Anar.

Lane-Garon, P.S., \& Richardson, T. (2003). Mediator mentors: Improving school climate, nurturing student disposition. Conflict Resolution Quarterly, 21, 47-67. https://doi.org/10.1002/crq.48

Lee, S., Kim, Ch.J., \& Kim, D.H. (2013). A meta-analysis of the effect of school-based anti-bullying programs. Journal of Child Health Care, 3, 1-18. https://doi.org/0.1177/1367493513503581

Lo Cricchio, M. G., García-Poole, C., te Brinke, L. W., Bianchi, D., \& Menesini, E. (2021). Moral disengagement and cyberbullying involvement: A systematic review. European Journal of Developmental Psychology, 18(2), 271-311. https://doi.org/10.1080/17405629.2020.1782186 
Menesini, E., Codecasa, E., Benelli, B., \& Cowie, H. (2003). Enhancing children's responsibility to take action against bullying: Evaluation of a befriending intervention in Italian middle schools. Aggressive Behavior, 29, 1-14. https://doi.org/10.1002/ab.80012

Menesini, E., \& Salmivalli, C. (2017). Bullying in schools: the state of knowledge and effective interventions. Psychology, Health \& Medicine, 22, 240-253. https://doi.org/10.1080/13548506.2017.1279740

Ortega, R., \& Del Rey, R. (2003). La violencia escolar: Estrategias de prevención. Graó.

Ortega, R., Del Rey, R., \& Mora-Merchán, J.A. (2004). SAVE model: an anti-bullying intervention in Spain. En P.K. Smith, D. Pepler \& K. Rigby (Eds.), Bullying in schools. How successful can interventions be? (pp. 167185). Cambridge University Press.

Palladino, B.E., Nocentini, A., \& Menesini, E. (2012). Online and offline peer led models against bullying and cyberbullying. Psicothema, 24, 634-639. [fecha de Consulta 23 de mayo de 2021]. ISSN: 0214-9915. Disponible en: https://www.redalyc.org/articulo.oa?id=72723959020

Rigby, K., \& Slee, P. T. (1991). Bullying among Australian school children: Reported behavior and attitudes toward victims. The Journal of Social Psychology, 131(5), 615-627. https://doi.org/10.1080/00224545.1991.9 924646

Romera, E., Ortega, R., del Rey, R., Casas, J. A., Viejo, C., Gómez, O., Córdoba, F., Zych, I., García, C., \& Luque, R. (2017). Bullying, cyberbullying y dating violence: Estudio de la gestión de la vida social en estudiantes de Primaria y Secundaria en Andalucía: Fundación Pública Andaluza.

Romera, E. M., Ortega-Ruiz, R., Runions, K., \& Falla, D. (2021). Moral disengagement strategies in online and offline bullying. Psychosocial Intervention, 30(2), 85-93. https://doi.org/10.5093/pi2020a21

Salmivalli, C., Lagerspetz, K., Björkqvist, K., Österman, K., \& Kaukiainen, A. (1996). Bullying as a group process. Participant roles and their relations to social status within the group. Aggressive Behavior, 22, 1-15. https:// doi.org/10.1002/(SICI)1098-2337(1996)22:1<1::AID-AB1>3.0.CO;2-T

Salmivalli, C., Kaukiainen, A., \& Voeten, M. (2005). Anti-bullying intervention: Implementation and outcome. British Journal of Educational Psychology, 75, 465-487. https://doi.org/10.1348/000709905X26011

Sánchez, V., Ortega, R., \& Menesini, E. (2012). La competencia emocional de agresores y víctimas de bullying. Anales de Psicología, 28(7), 71-82. [fecha de Consulta 23 de mayo de 2021]. ISSN: 0212-9728. Disponible en: https://www.redalyc.org/articulo.oa?id=16723161009

Silva, I., Torrego, J. C. \& Lorenzo-Llamas, E. M. (2021). Estudio de las estrategias de resolución de conflictos y habilidades sociales de alumnas y alumnos mediadores en educación secundaria obligatoria. South Florida Journal of Health, 2(7), 89-105.

Smith, P.K. (2003). Violence in schools. The response in Europe. Routledge Falmer.

Smith, P.K., \& Watson, D. (2004). Evaluation of the CHIPS (Childline in Partnership with Schools) programme. Research report RR570. DfES publications.

Sutton, J., \& Smith, P. K. (1999). Bullying as a group process: An adaptation of the participant role approach. Aggressive behavior, 25(2), 97-111. https://doi.org/10.1002/(SICI)1098-2337(1999)25:2<97::AIDAB3>3.0.CO;2-7

Tirado, R. y Conde, S. (2016). Análisis estructural de la gestión de la convivencia escolar en centros de buenas prácticas de Andalucía (España). Educación XX7, 19, 153-178. [fecha de Consulta 23 de mayo de 2021]. ISSN: 1139-613X. Disponible en: https://www.redalyc.org/articulo.oa?id=70645811006

Tofti, M., Farrington, D., Losël, F., \& Loeber, R. (2011). Effectiveness of school-based programs to reduce bullying: A systematic and meta-analytic review. Journal of Experimental Criminology, 7, 27-56. https://doi. org/10.1007/s11292-010-9109-1

Tognetta, L. R. P., Avilés, J.M. \& Sales, P. J. (2016). Bullying, un problema moral: Representaciones de sí mismo y desconexiones morales. Revista de Educación, 373, 9-34.

Torrego, J. C. (2005). Mediación de conflictos en instituciones educativas: manual para la formación de mediadores. Narcea. 
Torrego, J.C., \& Galán, A. (2008). Investigación evaluativa sobre el programa de mediación de conflictos en centros escolares. Revista de Educación, 347, 369-394.

Usó, l., Villanueva, L. \& Adrián, J. E. (2016). Impact of peer mediation programs to prevent bullying behaviours in secondary schools. Infancia y Aprendizaje, 39, 499-527. https://doi.org/10.1080/02103702.2016.11891 22

Yeager, D.S., Fong, C.J., Lee, H.Y., \& Espelage, D.L. (2015). Decline in efficacy of anti-bullying programs among older adolescents: Theory and three-level meta-analysis. Journal of Applied Developmental Psychology, 37, 36-51. https://doi.org/10.1016/j.appdev.2014.11.005 